\title{
Mini-access branch-first total arch replacement and frozen elephant trunk procedure-how I do it
}

\author{
John D. L. Brookes, Tristan D. Yan \\ Department of Cardiothoracic Surgery, Royal Prince Alfred Hospital, The University of Sydney, Sydney, NSW, Australia \\ Correspondence to: Professor Tristan D. Yan. Head of Minimally Invasive \& Robotic Cardiothoracic Surgery Program, Royal Prince Alfred Hospital \\ and Sydney Adventist Hospital, The University of Sydney, Sydney, NSW, Australia. Email: tristanyan@annalscts.com.
}

Submitted May 18, 2020. Accepted for publication May 25, 2020.

doi: 10.21037/acs-2020-fet-28

View this article at: http://dx.doi.org/10.21037/acs-2020-fet-28

\section{Clinical vignette}

A 70-year-old gentleman was referred for management of a saccular aneurysm of the distal aortic arch. His past history was relevant for smoking, hypertension and hypercholesterolemia. He initially presented with a hoarse voice over a nine-month period. Laryngoscopy revealed an immobile left vocal chord and subsequent computed tomography (CT) demonstrated a $5 \mathrm{~cm}$ saccular aneurysm on the inferior aspect of the distal aortic arch, likely resulting from the contained rupture of a penetrating ulcer. There was intramural thrombus within the aneurysmal sac and calcification in the distal arch. An endovascular intervention was contraindicated due to the lack of an adequate proximal landing zone.

\section{Surgical technique}

Prior to operation a spinal drain was inserted for cord protection. Following intubation, a Swan-Ganz catheter and a central venous line were inserted in the right internal jugular vein. Right radial and left femoral arterial monitoring lines were inserted under ultrasound-guidance. Nasal and intra-vesical temperature monitoring probes were placed.

Surgical access was via an upper mini-sternotomy to the left third intercostal space (a reverse "J" incision), which maximizes the surgical access to the aortic arch, compared to a right-sided mini-sternotomy. Extrapericardial dissection began with complete thymectomy and resection of the fat pads around the epi-aortic arch vessels. The innominate vein was slung with a vessel loop. The pericardium was opened to the level of the right atrium and three traction sutures were placed on each side to hitch the pericardium to the skin.

A multi-perforated venous cannula was introduced via the right femoral vein using Seldinger technique under trans-esophageal echocardiography (TEE) guidance. It was noted that the patient had a large patent foramen ovale. The central arterial cannulation strategy was modified by directing the cannula towards the aortic valve. This was to reduce the risk of 'sandblast-effect' of the inflow cannula on the clot-filled aneurysm if the cannula were pointed distally. The arterial line was split to provide separate cerebral and systemic perfusions.

Whilst the patient was cooled to $20{ }^{\circ} \mathrm{C}$ a branch-first approach was adopted. All epi-aortic vessels were delineated using a ball-tip diathermy and slung with individual vessel loops. First, the left subclavian artery (LSCA) was clamped and its origin was oversewn with two layers of 3-O Prolene suture. The LSCA was endarterectomized at its origin due to calcification and anastomosed to the distal limb of a Trifurcated graft (Vascutek Terumo, Inchinnan, UK) in an end-to-end fashion using a 5-O running Prolene suture. Then the left common carotid artery (LCCA) was debranched in the same fashion and anastomosed to the middle limb of the Trifurcated graft in an end-toend fashion while the brain was being perfused via the innominate artery. After completion of the LSCA and LCCA debranching, the left side of the brain was perfused through the Trifurcated graft, which allowed debranching of the innominate artery. Satisfactory cerebral saturations were maintained throughout the case.

Once the patient reached $20{ }^{\circ} \mathrm{C}$, an aortic cross-clamp was applied proximal to the arterial cannula. Diastolic arrest 
was achieved with antegrade Custodiol cardioplegia. Lower body circulation was terminated and the ascending aorta was transected just distal to the cross-clamp. The aortic arch was opened longitudinally to the level of Zone 2. Utmost care was taken not to disturb the extensive atheroma in the distal arch. To exclude the saccular aneurysm and distal arch atheroma, a 30/32/100 mm Thoraflex Hybrid graft (Vascutek Terumo, Inchinnan, UK) was selected, which allowed a $10 \mathrm{~cm}$ frozen elephant trunk (FET) to be fed distally beyond Zone 2 with a $5 \mathrm{~cm}$ overlap with the nonaneurysmal segment of proximal descending aorta. This Thoraflex hybrid graft excluded the complex distal arch pathology and enabled an anastomosis to be performed with better quality aortic tissue in Zone 2, even via a mini-access incision. Subsequently, a perfusion cannula was inserted into the side-branch of the Thoraflex graft for distal organ perfusion. The patient was then rewarmed towards $37^{\circ} \mathrm{C}$.

An end-to-end anastamosis was created between the Thoraflex graft and the sino-tubular junction using 3-0 Prolene sutures. A side-biter clamp was utilized and a $1.5 \mathrm{~cm}$ incision was made in the Thoraflex graft just above the proximal anastomosis. The Trifurcated graft was then anastomosed in an end-to-side fashion.

The patient weaned well from cardiopulmonary bypass. Excellent haemostasis was achieved. The mini-sternotomy was closed with three interrupted double stainless steel wires and the overlying soft tissues were closed in layers.

\section{Comments}

A branch-first approach allows continuous cerebral perfusion, efficiently utilizes surgical time during the cooling period and enables step-wise exposure of the aortic arch. Zone 2 anastomosis for FET offers three main advantages: first, it minimizes injury to the recurrent laryngeal nerve. Second, it avoids manipulation of distal arch aneurysm and atheroma. Third, the anastomosis is technically simpler and ensures better hemostasis $(1,2)$.

Management of the LSCA is the Achilles' heel of total aortic arch replacement. LSCA can be anastomosed in an end-to-end fashion described above. Alternate approaches include extra-anatomic bypass between LSCA and LCCA or an end-to-side graft to the LSCA through an infra-clavicular incision with the graft brought to the ascending aortic graft via the left chest (3). Each of these strategies must be weighed against the patient's anatomical requirements, pathology and appropriate access to LSCA. It is also important to allow some flexibility in the order of reimplantation of the epiaortic vessels depending upon the access and cerebral perfusion status.

\section{Acknowledgments}

None.

\section{Footnote}

Conflicts of Interest: The authors have no conflicts of interest to declare.

Open Access Statement: This is an Open Access article distributed in accordance with the Creative Commons Attribution-NonCommercial-NoDerivs 4.0 International License (CC BY-NC-ND 4.0), which permits the noncommercial replication and distribution of the article with the strict proviso that no changes or edits are made and the original work is properly cited (including links to both the formal publication through the relevant DOI and the license). See: https://creativecommons.org/licenses/by-nc-nd/4.0/.

\section{References}

1. Matalanis G, Shi WY. An Australian experience with aortic arch replacement: a novel approach without circulatory arrest or deep hypothermia. Heart Lung Circ 2011;20:163-9.

2. Tian DH, Wan B, Di Eusanio M, et al. A systematic review and meta-analysis on the safety and efficacy of the frozen elephant trunk technique in aortic arch surgery. Ann Cardiothorac Surg 2013;2:581-91.

3. Hage A, Ginty O, Power A, et al. Management of the difficult left subclavian artery during aortic arch repair. Ann Cardiothorac Surg 2018;7:414-21.
Cite this article as: Brookes JDL, Yan TD. Mini-access branch-first total arch replacement and frozen elephant trunk procedure-how I do it. Ann Cardiothorac Surg 2020;9(3):246247. doi: 10.21037/acs-2020-fet-28 